\title{
The Wireless Image Monitoring System based on the Technology of nRF905 and WiFi
}

\author{
Zhang $\mathrm{Fu}^{*}$, Zhang Yakun, Wang Jun, Qiu Zhaomei, Mao Pengjun, Wang wei and Liu Hongmei \\ College of Agricultural Engineering, Henan University of Science and Technology, Luoyang, 471003, China
}

\begin{abstract}
The wireless image monitoring system in pig house was designed to solve the high costs and the low efficiency problem of pig house security management based on the technology of nRF905 and WiFi. The union of wireless multimedia sensor networks and Internet was realized by the WiFi technology. The performance of the wireless multimedia sensor of the pig house monitoring system was analyzed. The results showed that environment parameter information and the growth conditions of the monitoring area were monitored accurately and transmitted reliably by the pig house monitoring system, which will provide the research foundation and methods to the design of the wireless multimedia image data transmission.
\end{abstract}

Keywords: A wireless multimedia sensor networks, communication distance, packet loss rate, pig house.

\section{INTRODUCTION}

A large number of sensor nodes of wireless sensor networks were distributed randomly. The data, which was acquired by sensor nodes, was collected and transferred in unsupervised environments [1-4]. With the growing complexity of monitoring environment, the wireless sensor network, which has a function of simple data acquisition, could not meet the comprehensive monitoring of the physical environment. Based on the wireless sensor networks, the wireless multimedia sensor networks (WMSN) had the function of collecting multimedia information such as audio, video, images and so on. The fine-grained and accurate information monitoring of environmental information could be realized by WMSN [5-8].

The pig house environmental parameters are crucial to the pig growth. Currently, most of the piggery monitoring equipment, which were composed of cables, cable and camera, had the disadvantage of high cost erection, complex wiring, circuit aging and breaking risk [9-11]. Aiming at the present problem of piggery monitoring system, the union of lower the transmission spectrum of $430 \mathrm{MHz}$ and WiFi technology was introduced into the wireless multimedia sensor node. Compared with the existing wireless multimedia sensor technology, the Internet real-time monitoring of environment parameters and pig growth was realized by the union of image data transmission and remote monitoring technology.

\section{THE SYSTEM OVERALL DESIGN}

For the application environment of pig monitoring system, the practical application problems of acquisition and communication links were considered.
Wireless transmission network center base station and wireless base station were established respectively at dispatch monitoring center and each pig house. Catering to the monitoring need, several supervision points were set in the pig house, which were used to gather and process the monitoring information, such as videos and datum. The collected monitoring information was sent to the dispatch monitoring center through the wireless transmission link. The designed nodes shown in Fig. (1) in the research, which were used to monitor the pig house environmental information and the pig growth condition in remote way. The design of the system perception layer was consisted of the design of the sensor nodes and sink node. In view of the application demand, the characteristic of low-power, low-cost, extensible features and monitoring a variety of environmental parameters should be realized by the sensor node. As for transfer station of the whole system, the sink node had not only the function of data gathering and forwarding, but also the ability of forming the whole network. Various environmental parameters information was collected by sensor nodes within the monitoring area, and sent to the sink node by means of Ad-Hoc Network. At last the collected information was delivered to the remote server by the sink node via Internet. As a lot of radio interference was exist in the $2.4 \mathrm{GHz}$ frequency band, the $433 \mathrm{MHZ}$ band was used.

\section{THE NODE HARDWARE DESIGN}

The basic component unit of pig house monitoring system was sensor node which included the function of environmental factor collection, data processing and wireless communication [12]. Under the background of pig house environmental monitoring application, the wireless sensor node hardware was designed in this study. The structure diagram and the circuit diagram of multimedia monitoring node were shown in Figs. (2) and (3) respectively. 


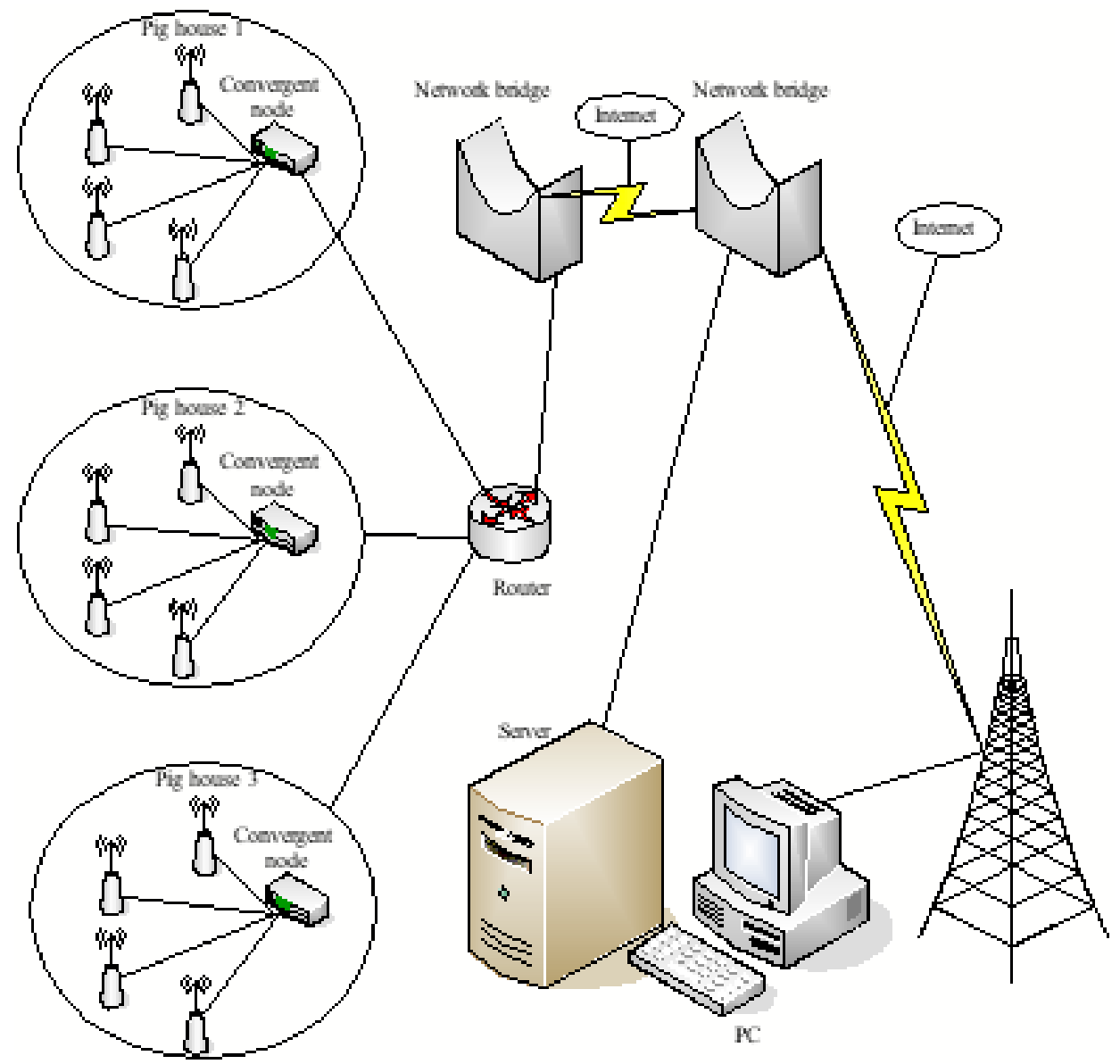

Fig. (1). The overall structure.

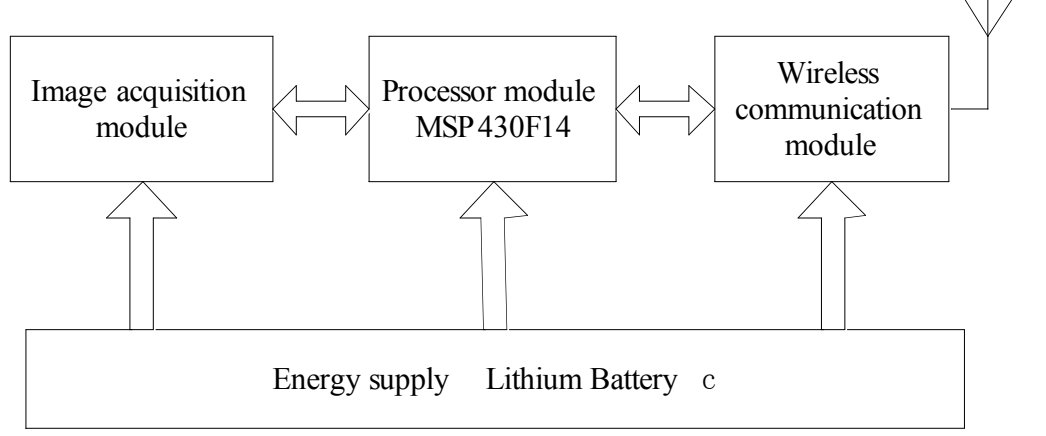

Fig. (2). Diagram of multimedia monitoring node.

\subsection{Microprocessor of Sensor Nodes}

Microprocessor module was the core part of sensor nodes, which had the responsible for regulating the other modules to complete the function of data collection, processing and transmission, etc. MCU MSP430F149, which was produced by TI Company, had rich inside peripheral set, was an ultra-low power 16-bit MCU, was advantageous for simplifying circuit and node size reducing. Its operating voltage was $1.8-3.6 \mathrm{~V}$. The consumption of RAM data storing was only $0.1 \mu \mathrm{A}$. The current consumption of low power mode was only $1.6 \mu \mathrm{A}$. The current of operation mode was as low as $280 \mu \mathrm{A}$, and the wake-up time from standby mode was less than $6 \mu \mathrm{s}$.

\subsubsection{Wireless Transmission Unit}

The nRF905 of Norwegian Nordic Company was selected to achieve the short-range wireless communications. As a radio frequency module, nRF905 provided the physical layer send and receive data transmission function, which reduced the energy consumption significantly. The working ISM band was $433 \mathrm{MHz}$. The output power and communication channels could be set through program.

\subsubsection{The Multimedia Sensor}

The industrial image acquisition and processing module PTC08 was selected as the multimedia sensor, which has the function of image acquisition, shooting control, data 


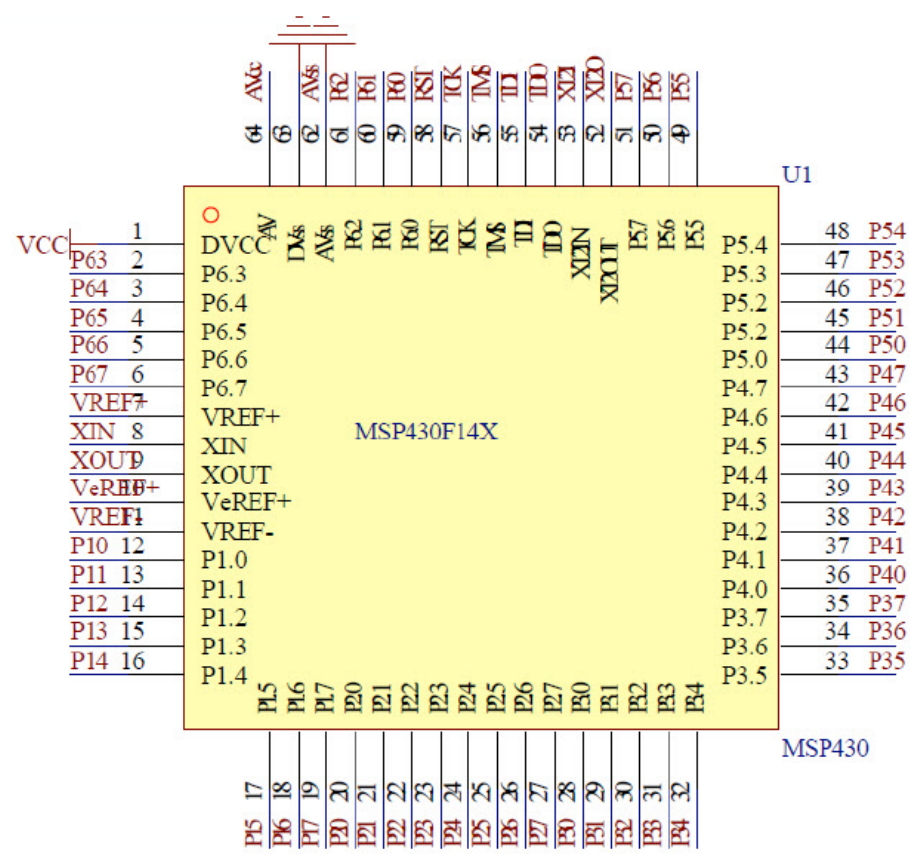

(a) MSP430 CPU

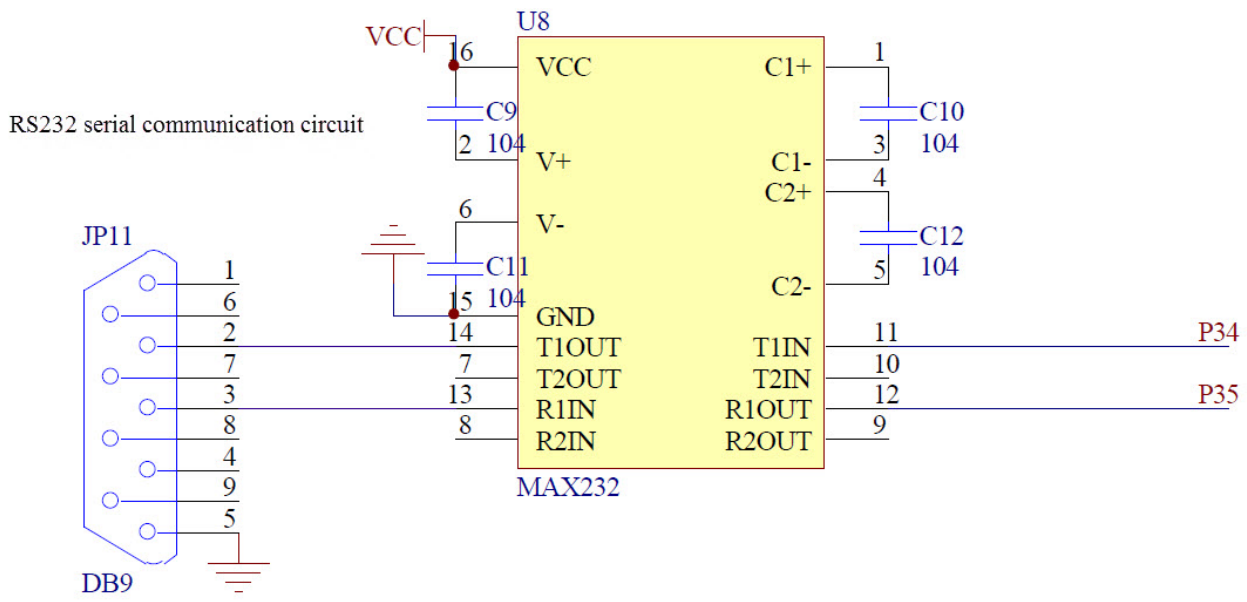

(b) RS232 serial communication circuit

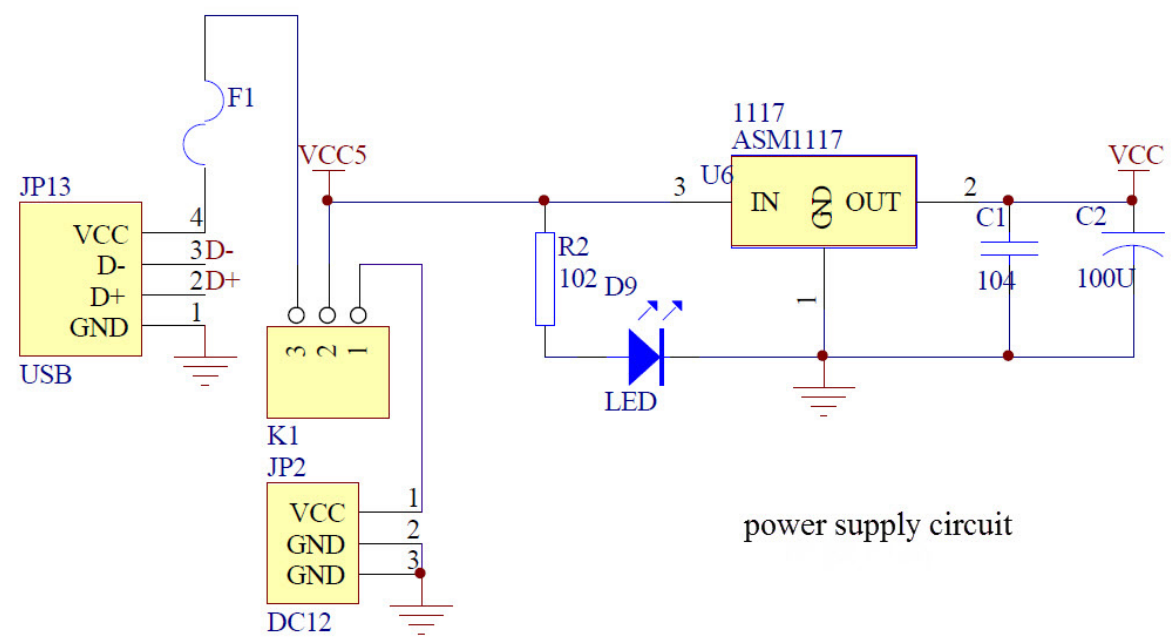

(c) Power supply circuit

Fig. (3). the circuit diagram of multimedia monitoring node. 


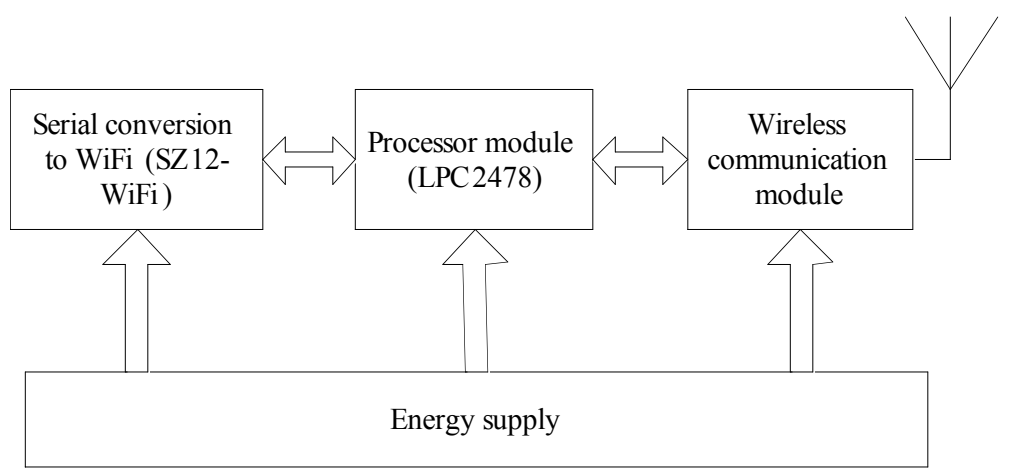

Fig. (4). Diagram of multimedia sink node.

compression and serial transmission. In order to reduce the transmission image data, the built-in high-performance digital signal processing chip was used to compress the original image in a high proportion. The standard RS-232 communication interface was adopted in the PTC08 to monitor environmental data. A variety of image data transmission rate was available with a flexible optional baud rate of 9600 , 19200, 38400, 57600 and 115200 .

\subsection{The Sink Node}

As the data convergence center, the sink node connected to the sensor network and finished the data convergence, and it uploaded the collected data to the remote server and issued the control commands while acting as a gateway device at the same time. On the one had, the underlying sensor node data was sent to the external network by serial-to-WIFI sent after a certain processing. On the one hand, the control command from the remote management center received by WiFi was dispatched to the whole sensor network. The Diagram of multimedia sink node as shown in Fig. (4).

\subsubsection{Microprocessor of Sink Node}

The sink node should have the stronger ability of processing, computing and communications. Since the simple function and slow data processing of a common 8-bit or 16bit microcontroller could meet the application need, LPC2478 produced by the PHILIPS Company, which had the ARM7 Core, was selected as the processor module of the sink node. The high-performance ARM7TDMI-S core was used, which had the characteristic of operating fast, rich in resources, good scalability and low power consumption.

\subsubsection{The Module of Serial Port to WiFi}

SZ12-WIFI WiFi module, which was produced by Shanghai Shun Zhou Electronic Technology Co, embedded the WiFi wireless networking standard based on the UART interface. The IEEE802.11 protocol stack of wireless networking protocol and TCP/IP protocol stack were built-in it. The data between serial port and the wireless network could be transformed easily.

\subsection{Program Design of Node Low-Power Management}

In the application of wireless sensor network technology, the energy was the key issue to limit its development. Therefore, at the design process of sensor node design, the low power requirements of the selection of hardware must be met, and low power management program design must be implemented in the software design. In the design of the program, the sleep of each module and the node wake cycles were controled by program. The process of program design was shown in Fig. (5). According to the practical requirements, the data was collect by sensor node at a certain sampling interval. During the time without data acquisition and transmission, the sensor nodes went into the sleep state. When it came to the sampling time, the processor was waked up by timer interrupt, followed by the work of the collection and transmission of various sensor data. After the design of a series of programming, the node image was taken in the remote control and the pig house environmental information and pig growth was monitored at a remote distance.

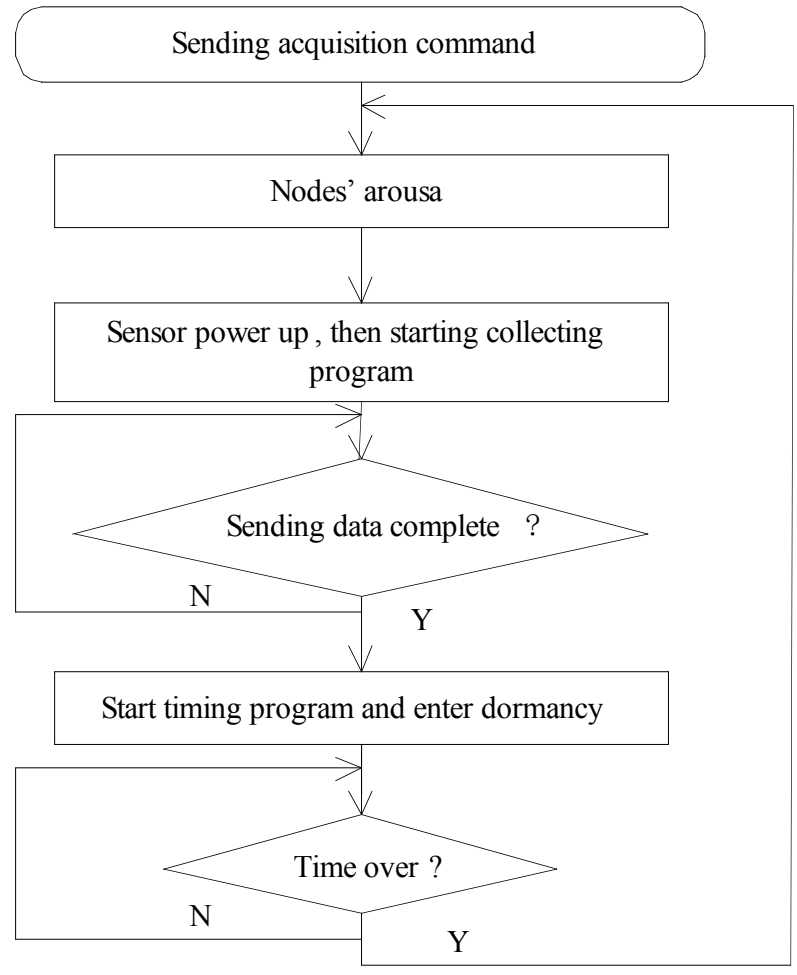

Fig. (5). Program flowchart of Node low-power management.

\section{THE SYSTEM TEST}

The link quality of sensor nodes was affected by the radio interference, the shape of the antenna, azimuth and distance and environmental factors, which may change with the 


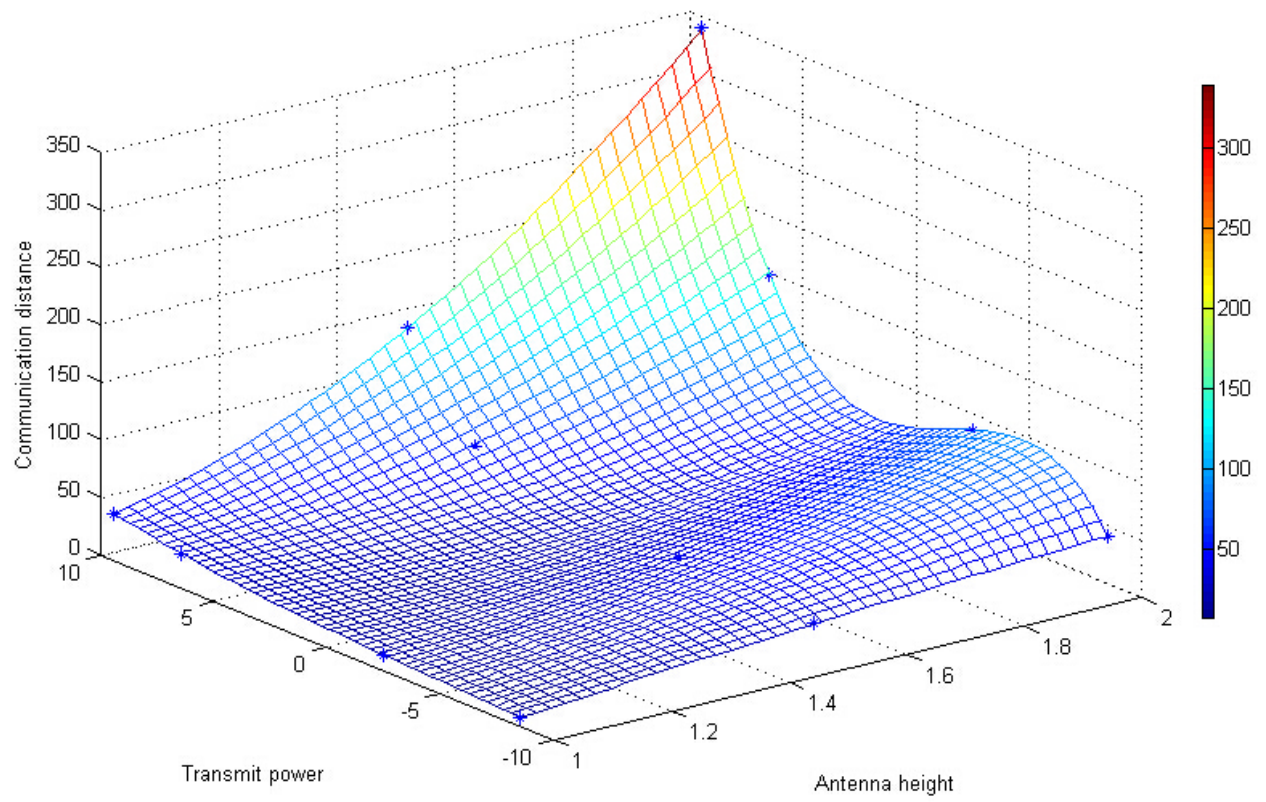

Fig. (6). Effect of the communication distance for different heights and the transmit power.

use of wireless sensor networks in wireless multimedia sensor networks [13].

\subsection{The Relationship Between the Height of Communica- tion, Transmission Power and Communication Distance}

In order to reduce the node energy consumption and prolong the life cycle, the communication quality, which was affected by the node communication height and the transmitting power, was discussed. Three antenna height of $1.0 \mathrm{~m}$, $1.5 \mathrm{~m}$ and $2 \mathrm{~m}$ and four transmit power of $10 \mathrm{dBm}, 6 \mathrm{dBm}$, $2 \mathrm{dBm}$ and $-10 \mathrm{dBm}$ were selected to conduct the experiment of communication distance. 1000 date packets were transmitted to the sink node each time. The communication distance of the antenna height and the transmit power were recorded when the average packet loss rate was more than $5 \%$. The result was shown in Fig. (6). From Fig. (6) we learned that with the increase of transmission power the communication distance increased under the same antenna height. The maximum communication distance and the minimum communication distance of nRF905 module were $40 \mathrm{~m}$ and $7 \mathrm{~m}$ respectively when the height of the antenna is $1 \mathrm{~m}$. The maximum communication distance and the minimum communication distance of nRF905 module were $140 \mathrm{~m}$ and $27 \mathrm{~m}$ respectively when the height of the antenna is $1.5 \mathrm{~m}$. At the same transmission power, the communication distance increased with the rise of antenna height.

\subsection{The Relationship Between the Packet Loss Rate and the Node Voltage}

The experiment conditions of the $1 \mathrm{~m}$ antenna height, $30 \mathrm{~m}$ communication distance and $10 \mathrm{dBm}$ transmit was set, and 1000 data was sent by the acquisition node each time. Then the packet loss rate was recorded under different voltage. The Fig. (7) showed that the node acquisition voltage and the packet loss rate had an inverse relationship. The higher node acquisition voltage was, the lower the packet loss rate was, and the lower acquisition node voltage was,

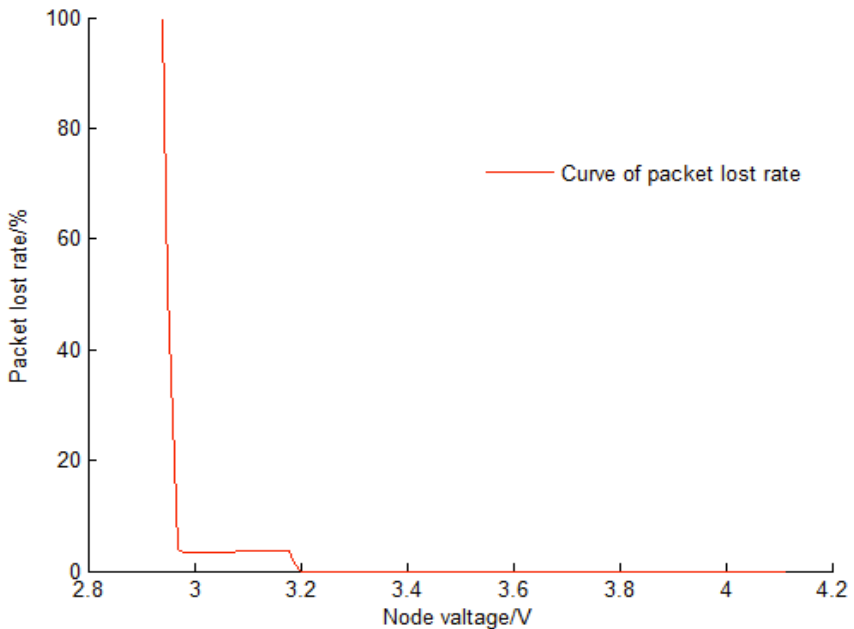

Fig. (7). The curve of packet loss rate under different voltage.

the higher the packet loss rate was. The packet loss rate was zero when the node acquisition voltage was between $4.11 \mathrm{~V}$ and $3.2 \mathrm{~V}$, but 1.2 percent when $3.19 \mathrm{~V}$. However, the packet loss rate was above $50 \%$ when the node acquisition voltage was below $2.95 \mathrm{~V}$. The node packet loss rate was less than 5 $\%$ when the node acquisition voltage was between $3.2 \mathrm{~V}$ and $2.97 \mathrm{~V}$. The application requirements could be satisfied when the node packet loss rate was less than $5 \%$. The multimedia sensor node communication went wrong when the packet loss rate was more than $5 \%$. So the node working voltage was as low as $2.97 \mathrm{~V}$.

\subsection{The Experiment of Pig House Environment}

The test was conducted in a fatting pig house, Mengjin County, Luoyang City, Henan Province, China. Four wireless multimedia sensor nodes and a sink node were placed. The placement height, transition power and baud rate were $1.5 \mathrm{~m},-9.5 \mathrm{dBm}$ and $38400 \mathrm{bps}$ respectively, and the communication module was set as 8 data bits, and no parity, 1 stop 
Table 1. The information under different communication distance.

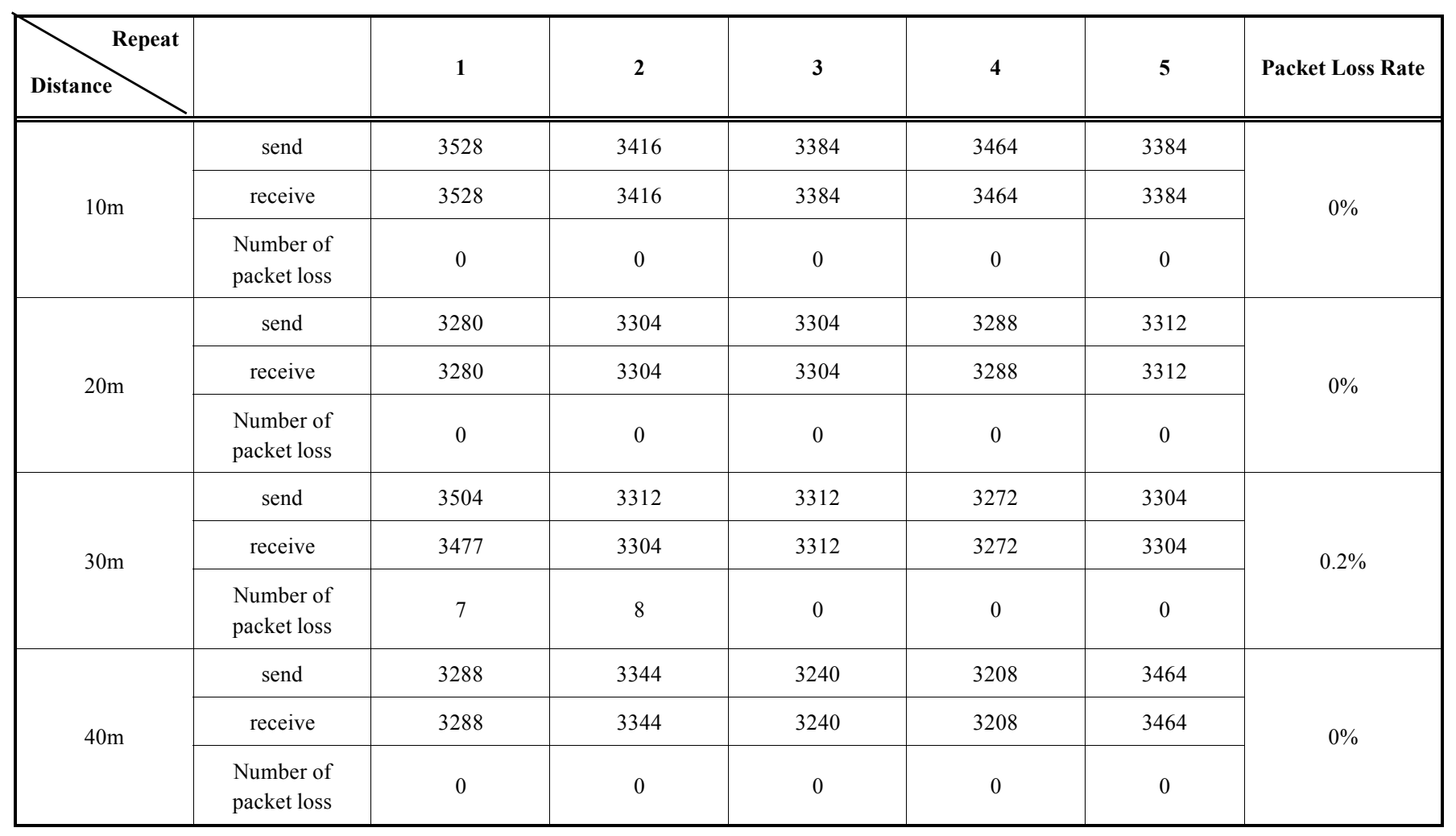

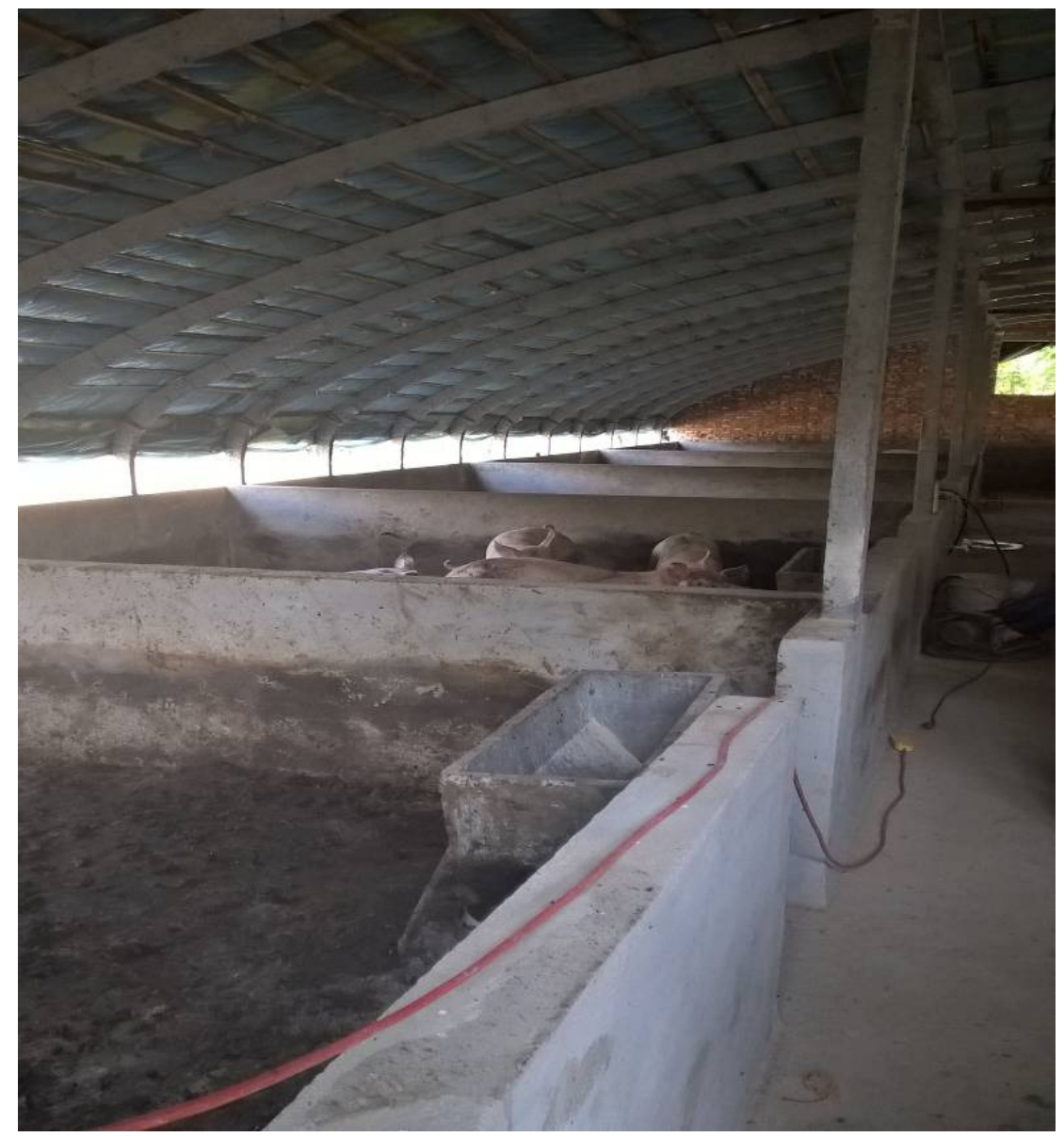

Fig. (8). The experimental scene of the pig house. 


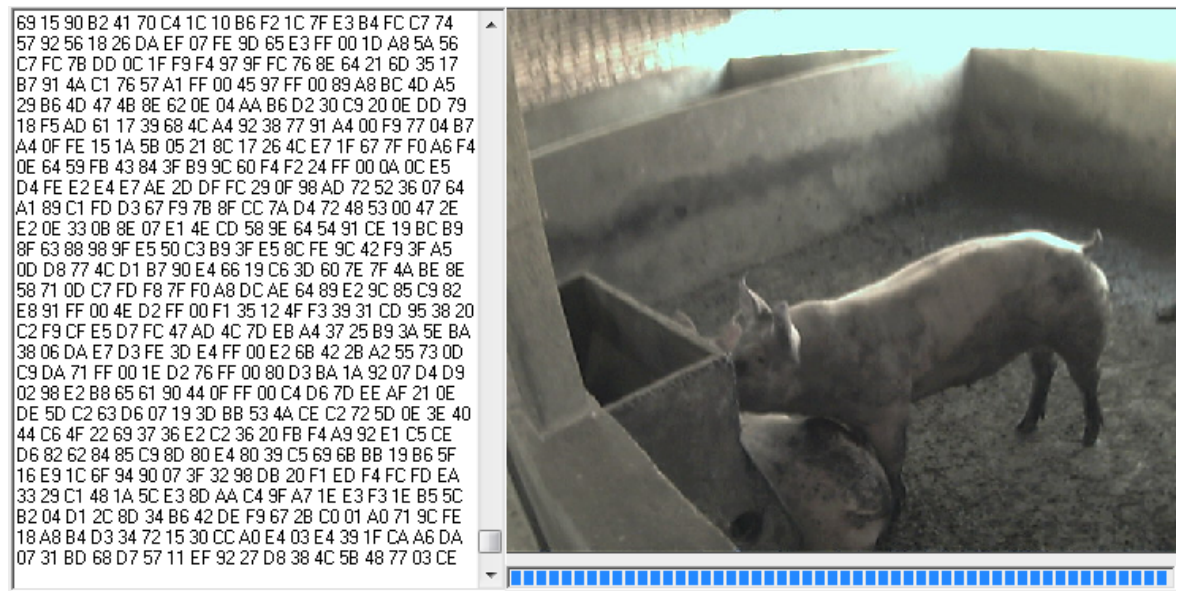

Fig. (9). The monitoring effect of wireless multimedia in pig house.

bit. The four wireless multimedia sensors were placed in the distance of $10 \mathrm{~m}, 20 \mathrm{~m}, 30 \mathrm{~m}$ and $40 \mathrm{~m}$ to the sink node. 5 pictures were taken in each wireless multimedia sensor, and the information was shown in Table $\mathbf{1}$.

The Table 1 shows that the designed wireless multimedia sensor node had a stable performance in the actual pig house environment. With the increase of node communication distance, the gathered image information was complete. The wireless multimedia sensor node installed in $30 \mathrm{~m}$ had a packet loss rate of $0.2 \%$, which was caused by the piggery structure. At the same time a packet loss rate of $0.2 \%$ did not affect the integrity of multimedia information, therefore the monitoring system designed of this study was stable and reliable, which could work under a certain environmental interference.

The experimental scene of the pig house the monitoring effect of wireless multimedia in pig house was shown in Figs. (8 and 9) respectively.

\section{CONCLUSION}

(1) The Wireless image monitoring system in the pig house was developed, which was based on based on the technology of NRF905 and WiFi. In the research, the system structure and the design of hardware and software were introduced. By integrating $\mathrm{WiFi}$ technology into the wireless multimedia sensor network, the fusion of wireless multimedia sensor network and internet was realized.

(2) The performance experiment of Wireless multimedia monitoring system in the pig house was implemented. The results show that: with the increase of transmission power the communication distance increased under the same antenna height. At the same transmission power, the communication distance increased with the rise of antenna height.

(3) The packet loss rate was inversely proportional to the voltage in this article. Through the experiment we found that the lowest voltage of wireless multimedia sensor networks constructed was $2.97 \mathrm{~V}$.

\section{CONFLICT OF INTEREST}

We declare that we have no financial and personal relationships with other organizations or people that can inap- propriately influence the research, there is no professional or other personal interest of any nature or kind in any product, service or company that could be construed as influencing the position presented in the manuscript entitled "The wireless image monitoring system based on the technology of nRF905 and WiFi in the pig house".

\section{ACKNOWLEDGEMENTS}

The project was supported by Project on the Integration of Industry, Education and Research of Henan Province (Grant No. 142107000055), Natural Science Foundation of Henan Educational Committee (Grant No. 14B416004, 14A416002, 13A416264), Key Project of Henan Tobacco Company (HYKJ201316) and Innovation Ability Foundation of Natural Science (Grant No. 2013ZCX002) of Henan University of Science and Technology.

\section{REFERENCES}

[1] Li Jianzhong, Gao Hong. Survey on Sensor Network Research [J]. Journal of Computer Research and Development, 2008, 45(1): 115.

[2] Wen Tao, Hong Tiansheng, Li Zhen. Test of wireless sensor network radio frequency signal propagation based on different node deployments in citrus orchards [J]. Transactions of the Chinese Society of Agricultural Engineering (Transactions of the CSAE), 2010, 26(4): 211-215.

[3] Zhao Zenghua, Shi Gaotao, Han Shuangli, et al. A heterogeneous wireless sensor network based remote district high-voltage transmission line on-line monitoring system [J]. Automation of Electric Power Systems, 2009, 33(19): 80-84.

[4] Luo Wusheng, Zhai Yongping, Lu Qin. Study on Wireless Multimedia Sensor Networks [J]. Journal of Electronics \& Information Technology, 2008,30(6): 1511-1516.

[5] Ma Huadong, Tao Dan. Multimedia Sensor Network and Its Research Progresses [J]. Journal of Software, 2006, 17(9): 2013-2028.

[6] Sun Yan, Ma Huadong. The QoS Guarantee Problem for Wireless Multimedia Sensor Networks [J]. Acta Electronica Since, 2008, 36(7): 1412-1420.

[7] Chen Changying, Yang Xiuhong, Fu Yong, et al. Some key issues in wireless multimedia sensor networks and their advances [J]. Shandong Science, 2013, 26(3): 48-53.

[8] Li Ruiyao, Bai Guangwei, Shen Hang, et al. Event-Driven QoS Communication Protocol in Wireless Multimedia Sensor Networks[J]. Chinese Journal of Sensors and Actuators, 2014, 27(6): 807-813.

[9] GARBRIEL L C, OVIDIU L P. Integrating power quality sensors in a wireless network [J]. Proceeding of 27th International Spring Seminar on Electronics Technology: Vol 1, May 13-16, 2004, Sofia, Bulgaria .Piscataway, NJ, USA:IEEE, 2004:153-157. 
[10] Ji Bin, Zhu Huawei, Li Xianfeng, et al. Background subtraction for static camera in pigpen[J]. Application Research of Computers, 2010, 28(9):3585-3590.

[11] Liang Wanjie, Cao Jing, Fan Yan, et al. Environment monitoring system for swine house based on wireless sensor network[J]. Jiangsu Journal of Agricultural Sciences, 2013, 29(6):1415-1420.

[12] Guo Wenchuan, Cheng Hanjie, Li Ruiming, et al. Greenhouse monitoring system based on wireless senor networks [J]. Transac- tions of the Chinese Society for Agricultural Machinery, 2010, 41(7): 181-185.

[13] Jia Kejin, Qian Chunyang, Han Yu, et al. Design of Wireless Sensor Node in Cold Chain Transportation Monitoring[J]. Transactions of the Chinese Society for Agricultural Machinery, 2013, 44(2): 136-141.

Received: June 10, 2015

Revised: July 29, 2015

Accepted: August 15, 2015

(C) Fu et al.; Licensee Bentham Open.

This is an open access article licensed under the terms of the (https://creativecommons.org/licenses/by/4.0/legalcode), which permits unrestricted, noncommercial use, distribution and reproduction in any medium, provided the work is properly cited. 